\title{
Interference Aware Dynamic Subchannel Allocation in a Multi-cellular OFDMA System Based on Traffic Situation
}

\author{
Banani Roy ${ }^{1}$, Chanchal Kumer Roy ${ }^{1}$, and Michael Einhaus ${ }^{2}$ \\ ${ }^{1}$ Queen's University, Kingston, Ontario, Canada K7L3N6, \\ $\{$ broy, croy\}@cs . queensu.ca \\ ${ }^{2}$ RWTH Aachen University, Aachen, Germany
}

\begin{abstract}
This paper presents the development and evaluation of a dynamic subchannel allocation scheme for downlink multi-cellular Orthogonal Frequency Division Multiple Access (OFDMA) systems. In the considered system each Access Point (AP) and the associated Mobile Terminals (MTs) are not operating on a frequency channel with a fixed bandwidth, rather the channel bandwidth for each AP is dynamically adapted according to the traffic load. The subchannels assignment procedure is based on quality estimations due to the interference measurements and the current traffic load. The developed dynamic subchannel allocation ensures Quality of Service (QoS), better traffic adaptability and higher spectrum efficiency with less computational complexity.
\end{abstract}

\section{Introduction}

Orthogonal Frequency Division Multiplexing (OFDM) is a multiple carrier technique that has been proved to be robust for communication over fading channels. OFDM systems divide a broadband channel into many narrowband orthogonal subcarriers $[8$, 3] where a group of subcarriers forms subchannels. When multiple accesses are desired OFDM can be combined with Frequency Division Multiple Access (FDMA) or Time Division Multiple Access (TDMA) or a mix of both. The combination of OFDM and TDMA is called Orthogonal Frequency Division Multiple Access (OFDMA). OFDMA is being considered as a modulation and multiple access method for the $4^{\text {th }}$ generation wireless networks. In OFDMA systems, radio resource allocation is still a critical issue in optimizing the performance of the system since the rapid increase in the size of the wireless mobile community and its demand for high speed multimedia communications stand in clear contrast to limited spectrum resources that have been allocated in international agreements. A feasible or nearly optimal dynamic subchannel allocation technique can be used for the resource allocation of an OFDMA system in order to optimize the performance. The motivation of this work is to allocate OFDMA subchannels to APs in a centrally controlled manner using Dynamic subChannel Allocation (DCA) scheme with effective frequency reuse by removing the inter cell interference remarkably. The newly developed DCA 
scheme works better than the Fixed subChannel Allocation (FCA) scheme with optimal Fixed Reuse Partitioning (FRP) [6] and the complexity of the algorithm is $\mathrm{O}\left(n^{2} \cdot m\right)+\mathrm{O}\left(k \cdot n^{2}\right), n$ is number of cells, $k$ is number of subchannels, $m$ is the number of users in the system.

The remainder of the paper is organized as follows. In Section 2 some previous works related to DCA scheme in OFDMA systems are presented. Section 3 discusses the developed DCA scheme, whereas the algorithmic complexity of this scheme is analyzed in Section 4. Section 5 briefly describes the deployed OFDMA system. The simulation results obtained from this system is provided in Section 6 and finally, Section 7 concludes the paper with some potential guidelines for further enhancement of this work.

\section{Previous Work}

Several DCA algorithms are developed, but their complexities are rather high [6]. In [12, the best channel reuse pattern was selected by doing the capacity prediction for all possible combination of the channel patterns. However, this type of solution is in practice impossible, especially where the number of cells and channels are many. Furukawa et al. [5], have mentioned that despite of the absence of intra-cell interference in OFDMA systems, optimum channel allocation is still a NP-hard problem which is basically complex. Moreover, individual user's rate requirements further complicate the problem. Kim et al. 7 and Pietrzyk et al. [11, have developed a channel allocation scheme based on [2] with less computational complexity. But in [11, a lot of iterations are needed to solve the problem and the linearization in 7 cannot be generalized to all types of modulations. Yin et al. 14, have investigated non-iterative algorithms to reduce computational complexity.

\section{Developed DCA Scheme}

In the developed subchannel allocation scheme traffic load is estimated based on the utilization of the assigned subchannels and the interference estimation is done based on the received signal power measurements. For this, a Hierarchical Radio Resource Management scheme of an OFDMA system has been designed by introducing an Access Point Controller (APC). The APC aims to handle inter cell interference and to allocate subchannels to the APs dynamically. Its functionalities are divided into two phases: reuse decision phase and allocation phase. In the first phase, the APC gains knowledge about the interference situation by investigating the mutual Signal to Interference Ratio (SIR) of the APs. In the second phase, the APC allocates subchannels to the APs using the obtained knowledge of interference. Two phases are discussed as follows:

\subsection{Reuse Decision Phase}

We have used the concept of Self Organizing Reuse Partitioning (SORP) [6], [5], but in a resource optimizing and centralized manner by exploiting the received 
signal power of the broadcast bursts in order to form the adaptive co-channel cells. This approach does not require any extra resources for calculating the mutual SIR. In this technique, the APC triggers the APs to measure the received signal power of the MTs periodically. After getting the invocation from the APC, the AP triggers its associated MTs to measure the received signal power level of the broadcast bursts. Then the associated MTs measure the received signal power level from all the APs and send the measured values to their controller AP. The controller APs then inform the APC about the received signal power values and the APC then estimates the mutual SIR of the APs using the received signal power values. Let us consider a small scenario with three APs: $A P_{0}, A P_{1}$ and $A P_{2}$. Each AP has four associated MTs. In this scenario the distance of $A P_{0} \& A P_{1}$ is $500 \mathrm{~m}, A P_{0} \& A P_{2}$ is $790 \mathrm{~m}, A P_{1} \& A P_{2}$ is $790 \mathrm{~m}$ and each cell radius is $200 \mathrm{~m}$ where cells are not overlapping. As $A P_{2}$ is far apart from $A P_{0}$ and $A P_{1}$, it can reuse resources of both $A P_{0}$ and $A P_{1}$. On the other hand, as $A P_{1}$ and $A P_{2}$ are not far apart, they have very low chance to reuse each other resources due to higher interference. The estimated mutual SIR values are shown in Table 1. The APC forms co-channel cells according to the mutual SIR values by comparing these with a predefined reuse threshold (here it is considered $15 d B$ ) and stores the binary reuse decisions: reusable $(y)$ and notreusable $(n)$ in a table called Reuse Partitioning Table (RPT). Different Reuse Constraints (RCs) can be used to form co-channel cells such as min mutual SIR, mean mutual SIR and weighted mutual SIR. Both in the example and simulation results, we have used

Table 1. Mutual SIR values for three-cell scenario

\begin{tabular}{|c||c|c|c|c|}
\hline \multirow{5}{*}{$A P_{0}$} & $M T_{0, i} / S I R$ & $A P_{0} / A P_{0}$ & $A P_{0} / A P_{1}$ & $A P_{0} / A P_{2}$ \\
\cline { 2 - 5 } & $M T_{0,100}$ & -- & 16.54 & 24.07 \\
& $M T_{0,101}$ & -- & 13.54 & 25.25 \\
& $M T_{0,102}$ & -- & $\underline{12.45}$ & $\underline{21.9}$ \\
& $M T_{0,103}$ & -- & 18.46 & 29.41 \\
\hline \hline \multirow{5}{*}{$A P_{1}$} & $M T_{1, i} / S I R$ & $A P_{1} / A P_{0}$ & $A P_{1} / A P_{1}$ & $A P_{1} / A P_{2}$ \\
\cline { 2 - 5 } & $M T_{1,104}$ & 16.13 & -- & 27.09 \\
& $M T_{1,105}$ & 13.53 & -- & $\underline{25.63}$ \\
& $M T_{1,106}$ & $\underline{12.39}$ & -- & 25.65 \\
& $M T_{1,107}$ & 18.68 & -- & 25.64 \\
\hline \hline \multirow{5}{*}{$A P_{2}$} & $M T_{2, i} / S I R$ & $A P_{2} / A P_{0}$ & $A P_{2} / A P_{1}$ & $A P_{2} / A P_{2}$ \\
\cline { 2 - 5 } & $M T_{2,108}$ & $\underline{24.07}$ & $\underline{24.43}$ & -- \\
& $M T_{2,109}$ & 33.03 & 33.41 & - \\
& $M T_{2,110}$ & 45.49 & 45.69 & - \\
& $M T_{2,111}$ & 33.12 & 33.29 & - \\
\hline
\end{tabular}

min RC, the minimum mutual SIR for all the MTs associated to an AP (for details see [13]). But it is not possible to remove the interference remarkably by considering merely this binary decision due to the indirect resource sharing. It 
has been noticed that when two APs form co-channel cells, another AP could be interfered if it can only be able to form co-channel cells with one of the two APs. The APC filters this type of indirect interference by finding out a conflicting set of APs where APs interfere each other by reusing other APs' resources. Then it updates the RPT by introducing another reuse decision: partially reusable $(p)$ for those conflicting APs by replacing the reusable decision. For example in the above mentioned scenario, $A P_{0} \& A P_{2}$ and $A P_{1} \& A P_{2}$ can form cochannel cells, but $A P_{0} \& A P_{1}$ cannot by using min RC (see Table 1 ). Thereby, if $A P_{0}$ and $A P_{1}$ use same resources of $A P_{2}$, they will interfere with each other. Consequently, the APC partially assigns $A P_{2}$ 's subchannels to $A P_{0}$ and $A P_{1}$
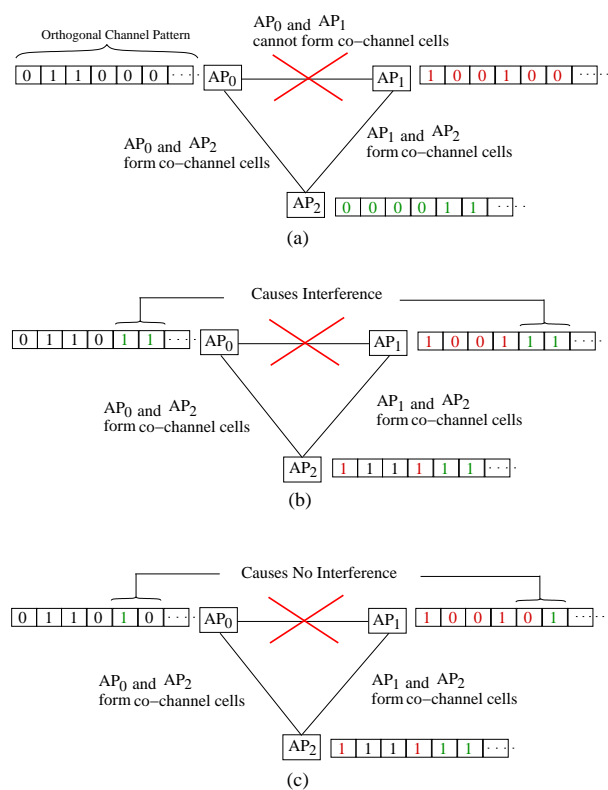

Fig. 1. Filtering interference

in an orthogonal manner which is shown in Fig. 11 The APC then assigns a weighted value to each decision where the reusable decision is assigned value 1 i.e. an AP can use whole resource of the interferer AP. The notreusable decision is assigned value 0 i.e. resources cannot be reused between two APs. The value for the partial decision is calculated based on the number of candidate APs using another AP's exclusive resources in an orthogonal manner; for example, in the scenario explained earlier, $A P_{1}$ and $A P_{2}$ will partially use $A P_{0}$ 's resources. As there are two APs to use $A P_{0}$ 's orthogonal subchannels, the value of the partial decision will be $1 / 2$. The sum of each AP's decision values called Reuse Decision Values (RDVs) are shown in Fig. 2] (bottom-right part). 


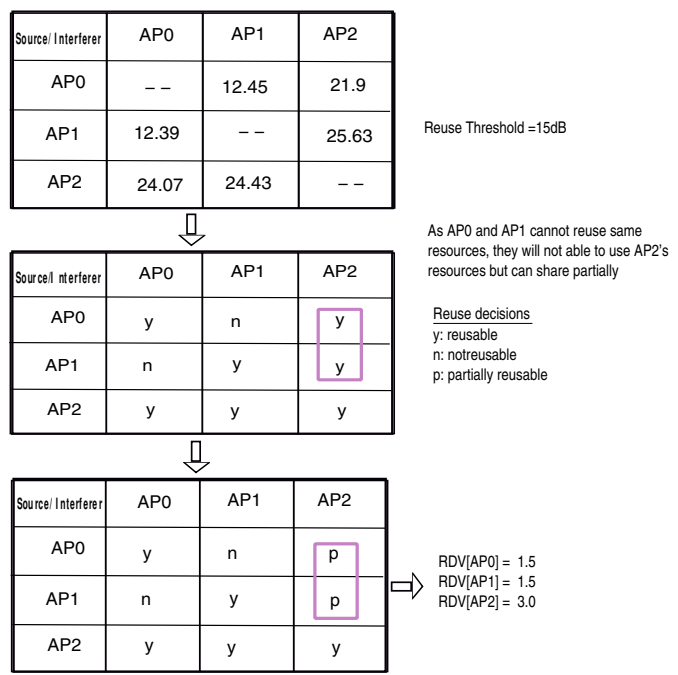

Fig. 2. Working steps of reuse decision phase

\subsection{Allocation Phase}

In this phase, the subchannel requirements of the APs are calculated by multiplying the value of the utilization function (weighted mean of the utilization [12, [13]) with the previously assigned subchannels of APs. The higher utilization function value indicates higher resource requirement for an AP. Then the priority level of an AP is calculated for the assignment of orthogonal subchannels by dividing the subchannel requirements with the corresponding RDV. The steps in the reuse decision phase are as follows:

- Calculate the required subchannels using estimated utilization and previously assigned subchannels

- Loop until all the subchannels are assigned (allocation of orthogonal subchannels)

- Calculate priority levels

- Select the AP with the highest priority

- Assign subchannel to the selected AP

- Reduce the priority level

- Distribute reusable subchannels based on the RPT

\section{Algorithm Complexity}

The complexity to calculate mutual SIR is $O\left(n^{2} \cdot m\right)$. Here two loops involve the $n$ number of APs associated to the APC and one loop involves $m$ number of MTs associated to an AP. The complexity of generating RDV matrix is $O\left(n^{2}\right)$ where 
two loops involve the $n$ number of APs associated to the APC. The complexity of the orthogonal subchannel allocation scheme is $O\left(k \cdot n^{2}\right)$ as it involves three loops. The outer most loop involves $k$ number of subchannels and the inner two loops involve $n$ number of APs. The complexity of distributing reusable subchannels is $O\left(n^{2} \cdot k\right)$ as it also involves two loops for the $n$ number of APs and one loop for $k$ subchannels. So the over all complexity of this algorithm can be written as $O\left(n^{2} \cdot m\right)+O\left(k \cdot n^{2}\right)$ which is better than previous works cited in Section 2.

\section{Deplyed OFDMA System Description}

The MAC structure is based on IEEE 802.16a, whereby the Physical Layer (PHY) differs significantly. The channel bandwidth of the system is $80 \mathrm{MHz}$ which is subdivided into 1024 subcarriers with a spacing of $78.125 \mathrm{kHz}$. Due to orthogonality, the total symbol length is $13.6 \mu \mathrm{s}$ including a guard interval of $0.8 \mu \mathrm{s}$ to mitigate inter symbol interference in multipath environment. An OFDMA subchannel consists of 32 subcarriers, whereby 2 subcarriers per subchannel are reserved for the transmission of pilot signals. The subchannels are directly mapped onto a contiguous fraction of the frequency channel. This is in contrast to IEEE 802.16a, where the subcarriers of a subchannel are evenly distributed over the frequency channel in a pseudo random manner to apply a kind of spreading scheme [8]. This certainly reduces the flexibility of the scheduling since the diversity of the subchannel is lost. Three different combinations of modulation schemes are used for the performance evaluation, namely QPSK 3/4, 16QAM 3/4 and 64QAM 3/4. These schemes are called PHY mode in this work. An important feature of the OFDMA PHY is the possibility of exploiting multi-user diversity. The general MAC structure is based on a centrally controlled scheme like IEEE 802.16. The frequency channel is divided into MAC frames. In this protocol, the frame length is calculated using the symbol length and the number of slots per frame. Here it is considered that the symbol length or duration is $13.6 \mu \mathrm{s}$ and symbols per slot are 8 . Slots could be taken as any number greater than 2 because one slot is reserved for the signaling overhead. The signaling overhead includes the transmission of control data packets. These packets comprise messages for channel estimation and resource allocation. The remaining slots are scheduled for downlink transmissions as uplink was beyond the scope of this work. Overall, a fixed signaling overhead of one time slot is assumed independent of the amount of used resources.

\section{Simulation results}

The simulation of this work is performed extending NS2 [9] whereby the statistical evaluation is conducted following 10. Here for the traffic source model, a two-state Markov Modulated Poisson Process (MMPP) 4 is used. The two-state MMPP is in either ON state or is in OFF state. In this model packets are only generated in the $\mathrm{ON}$ state with fixed arrival rate $\alpha$. The time spent in $\mathrm{ON}$ and 
OFF states is exponentially distributed with mean $\alpha^{-1}$ and $\beta^{-1}$ respectively. It is also called Interrupted Poisson Process (IPP) with fixed inter-arrival time. The position of the MTs is updated at each $1 \mathrm{~ms}$ whereby the displacement of the MTs is characterized by the Brownian motion mobility model [1. In this model MTs move in the adjacent segment with reflecting the mobility region and the position of the mobile user is always changed by $5 \mathrm{~m}$. The simulation results are presented in the subsequent subsections.

\subsection{Balancing Resource Utilization}

Before going to the performance analysis of newly developed DCA scheme, the correctness of the algorithm must be proved. In this regard, we have to show whether the algorithm is reacting on the various traffic situation or not. Reacting on various traffic load means that the APC will assign more subchannels in case of high traffic load situation than the case of low traffic load situation. In order to prove it, we have simulated a three cell circular scenario where each cell has 26 MTs and the radius of the mobility region is $300 \mathrm{~m}$. In the scenario, (Fig. (3) each AP has been assigned different traffic loads per downlink connections. The APC updates resources at the frame interval 10 while it updates the mutual SIR value at the frame interval 20. $A P_{0}$ is assigned load $3000 \mathrm{kbps}, A P_{1}$ is $500 \mathrm{kbps}$ and $A P_{2}$ is $10000 \mathrm{kbps}$. In this scenario, for the traffic load the mean on time is 0.1 and mean off time is 0.4, OFDMA subchannels are 32 and time slots are 17 . There is no reuse of subchannels as the spatial distances between the

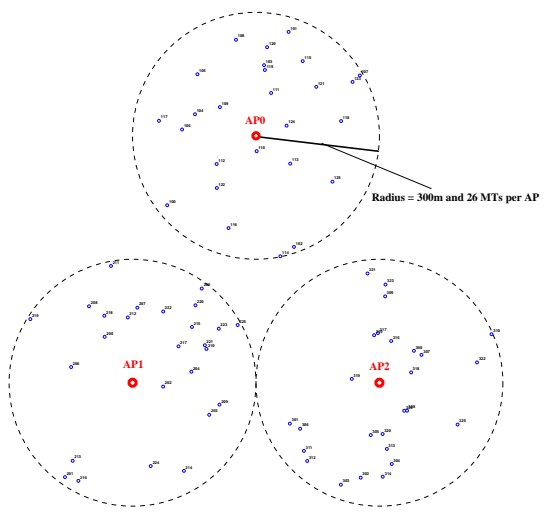

Fig. 3. Three cell scenario

APs are not sufficient for the resource reuse. The resource utilization curve is shown in Fig. 4(a) The $\mathrm{X}$-axis is for the utilization that ranges from $0-1$ and Y-axis is for the Complementary Cumulative Distribution Function (CCDF) of the corresponding utilization. The CCDF decreases with the increasing assigned 
utilization. In the curve on average $A P_{0}$ is utilizing $35 \%$ of the assigned resources, $A P_{1}$ is $18 \%$ and $A P_{2}$ is $37 \%$. These average values indicate that the APs are utilizing resources according to the assigned traffic loads. The APC has balanced the resource utilization of all the APs by assigning subchannels according to the traffic load. This effect can be seen in Fig. 4(b) In the curve the subchannels assignment to each AP with the corresponding traffic load is shown. As $A P_{2}$ has highest resource requirement, it has got the highest number of subchannels whereas as $A P_{1}$ has lowest resource requirement, it has obtained lowest number of subchannels. In case of $A P_{0}$, the number of subchannel assignment is in the middle position. It has obtained a higher number of subchannels than that of $A P_{1}$ and a lower number of subchannels than that of $A P_{2}$. So from analyzing the number of obtained subchannels and utilization with various traffic loads, we can say that the developed algorithm in this paper works correctly.

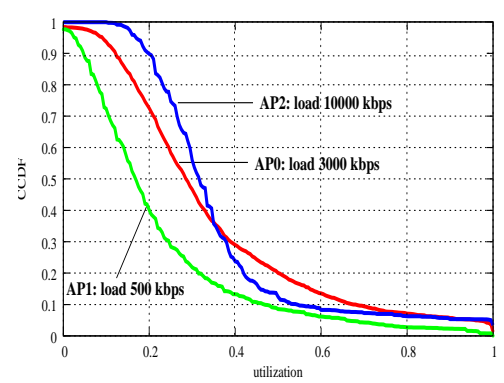

(a) Utilization

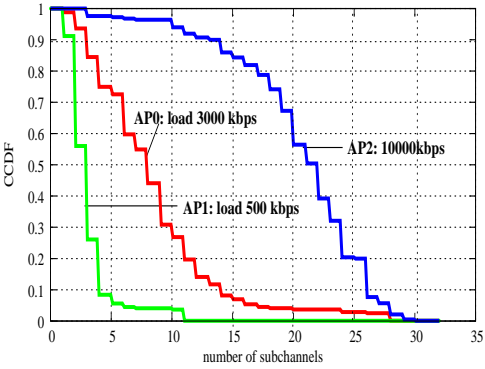

(b) Subchannels

Fig. 4. Resource utilization and subchannel assignment on various traffic load

\subsection{Finding Optimal FRP for a five cell scenario}

In order to compare the newly developed DCA scheme with the fixed FCA

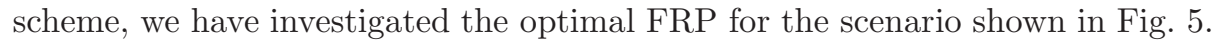
In this case, we have used 16 OFDMA subchannels, 10 MTs per cell and 9 time slots per subchannel and both mean on and mean off time are 0.1 in traffic load generation. We have obtained the optimal FRP for this scenario by investigating three feasible FRPs that are as follows:

$\operatorname{coset} A=\left\{A P_{0}, A P_{1}, A P_{3}, A P_{4}\right\} ;\left\{A P_{2}\right\}$, $\operatorname{coset} B=\left\{A P_{0}, A P_{3}\right\} ;\left\{A P_{2}\right\} ;\left\{A P_{1}, A P_{4}\right\}$,

$\operatorname{coset} C=\left\{A P_{0}\right\} ;\left\{A P_{1}\right\} ;\left\{A P_{2}\right\} ;\left\{A P_{3}\right\} ;\left\{A P_{4}\right\}$

In $\operatorname{coset} A$, the average number of subchannels assigned to each set is 8 , in $\operatorname{coset} B$ is 5.33 and in $\operatorname{coset} C$ is 3.2 . Here different simulations have been performed by 
varying the loads of $A P_{2}$ and $A P_{4}$ while keeping fixed the loads of $A P_{0}, A P_{1}$ and $A P_{3}$. In the corresponding simulation result curves, $\operatorname{coset} A$ will be termed as 2 co-channel cells because it has two clusters. Similarly, $\operatorname{coset} B$ will be 3 -co-channel cells sets and $\operatorname{coset} C$ will be 5 -co-channel cells sets. Finally, by simulating the same scenario with these three reuse partitioning we found that $\operatorname{coset} B$ is the optimal reuse partitioning in terms of mean delay. The simulation results show

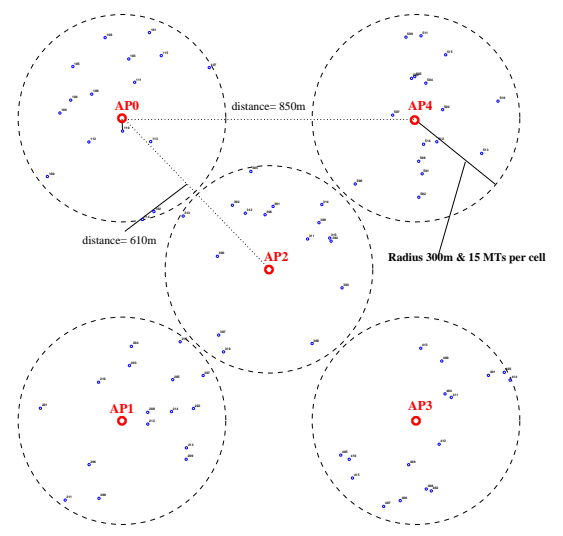

Fig. 5. Five cell scenario

that the mean delay of $\operatorname{coset} B$ is less than that of $\operatorname{coset} A$ and $\operatorname{coset} C$ shown in Fig. 6(a) as in $\operatorname{coset} A$, the co-channel interference increases by allowing $A P_{0}$, $A P_{1}, A P_{3}$ and $A P_{4}$ to reuse their subchannels; therefore, the Packet Error Rate (PER) increases (Fig. 6(b) which shows that the PER for the $\operatorname{coset} A$ is greater than the maximum allowable PER 0.01 in the considered system. For $\operatorname{coset} C$, the mean PER is less than 0.01 since each cell uses orthogonal subchannels, and consequently, there is less co-channel interference.

Although in case of coset $B$ mean PER is higher (there is reuse of subchannels) than $\operatorname{coset} C$, mean delay decreases on the higher traffic loads for the better utilization of the resources than the $\operatorname{coset} C$. In case of $\operatorname{coset} C$, delay increases on the higher traffic loads due to the higher resource requirement of $A P_{2}$ and $A P_{4}$. As they do not get more subchannels on increasing load, the packets are queued, and therefore, the mean delay increases. The mean utilization curve is shown in Fig. 6(c) The mean utilization is around $50 \%$ because different simulations have been performed only increasing the load for two $\operatorname{APs}\left(A P_{2}\right.$ and $\left.A P_{4}\right)$ by keeping others fixed. Here the utilization of the APs whose loads were fixed is too low and thereby, the mean utilization decreases to $50 \%$. The curve shows that the utilization for $\operatorname{coset} C$ is the highest, and with the increasing traffic load it becomes saturated due to the limited queue length. But in case of $\operatorname{coset} A$, the mean utilization is upward at the high load as in this case packets retransmission is higher for the co-channel interference. 


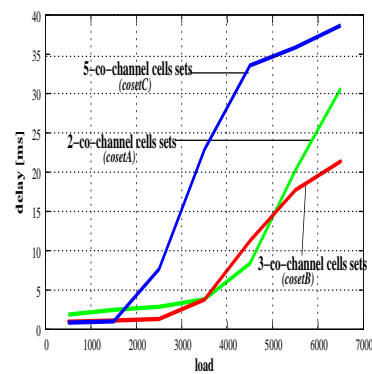

(a) Mean delay

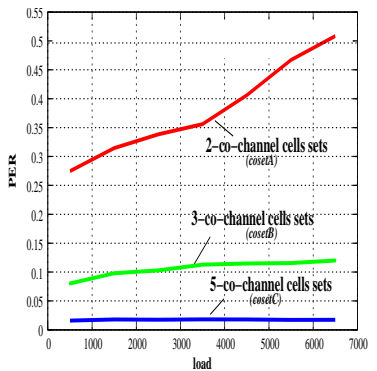

(b) Mean PER

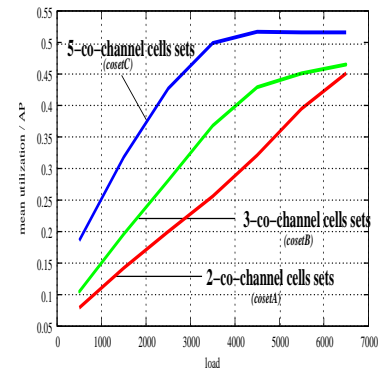

(c) Mean utilization

Fig. 6. Comparison of mean utilization, PER and delay among 3 FRPs

\subsection{Comparison between developed DCA scheme and FCA scheme}

In this subsection, we compare the performance of the newly developed Dynamic subChannel Allocation (DCA) scheme with Fixed subChannel Allocation scheme

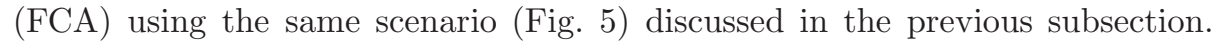
Here for the FCA scheme the optimal cell FRP $\operatorname{coset} B$ (discussed in the above subsection) has been used. In this partitioning, co-channel set $\left\{A P_{0}, A P_{3}\right\}$ gets 6 subchannels while the remaining two co-channel cells sets, $\left\{A P_{1}, A P_{4}\right\}$ and $\left\{A P_{2}\right\}$ get 5 subchannels at each. As DCA scheme always performs better than FCA scheme in an uneven traffic situation, we have done different simulations with increasing traffic load for $A P_{2}$ and $A P_{4}$ while keeping fixed the traffic loads of $A P_{0}, A P_{1}$ and $A P_{3}$ at $500 \mathrm{kbps}$. As a result, different resource requirement behaviors were prevailed in the APs. The mean utilization of resources for each of the APs is shown in Fig. 7(a) In case of the FCA, in the mean utilization curves, $A P_{0}, A P_{1}$ and $A P_{3}$ have always a lower utilization as their traffic load is fixed at 500kbps. Their average utilization is around $10 \%$. On the other hand, $A P_{2}$ and $A P_{4}$ are over loaded in case of higher traffic load. Their utilization is greater than $80 \%$ after the load $3500 \mathrm{kbps}$. Whereas in the DCA, $A P_{0}, A P_{1}$ and $A P_{3}$ utilize $50 \%$ of the assigned resources on average and $A P_{2}$ and $A P_{4}$ utilize $70 \%$ of the assigned resources on average. The mean delay for the FCA scheme is greater than that of the DCA scheme (shown in Fig. 7(b) as the FCA scheme is not assigning sufficient subchannels to $A P_{2}$ and $A P_{4}$ on increasing load. Another reason is that the PER increases as the FRP of cells is used by the FCA scheme which is shown in Fig. 7(c) But in case of the DCA scheme, as adaptive cell reuse partitioning is used, the mean PER is much lower than the FCA. The mean number of assigned subchannels for each the AP is shown in Fig. 7(d) As in the used scenario the APs are not sufficiently far apart to reuse resources and the loads of $A P_{2}$ and $A P_{4}$ are varying, both are getting higher number of subchannels on increasing load. On the other hand, as the loads of the remaining three $\mathrm{APs}\left(A P_{0}, A P_{1} \& A P_{3}\right)$ are fixed, they are getting lower number of subchannels with increasing loads of $A P_{2}$ and $A P_{4}$. 


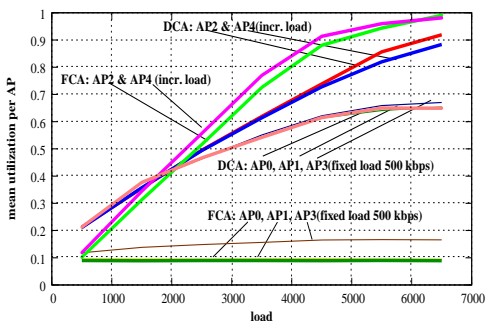

(a) Mean utilization

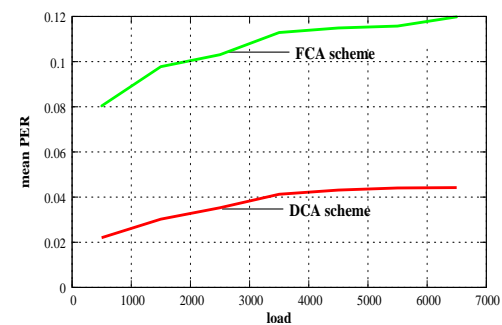

(c) Mean PER

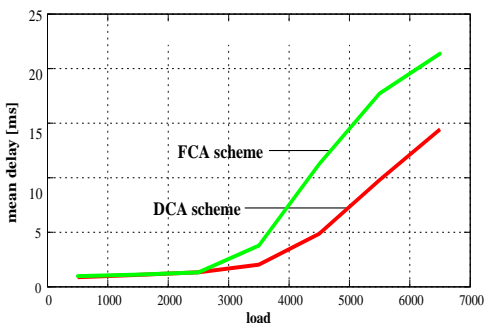

(b) Mean delay

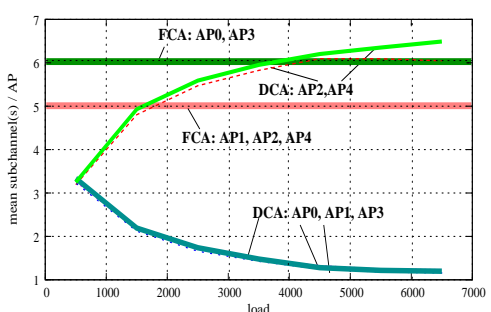

(d) Mean subchannels/AP

Fig. 7. Mean delay comparison between DCA and FCA schemes on increasing load of $A P_{2}$ and $A P_{4}$ and fixed load of $A P_{0}, A P_{1}$ and $A P_{3}$

\section{Conclusion and future work}

This work shows that the intelligently handled interference aware DCA scheme has a great benefit compare to FCA schemes in case of uneven traffic situation. This is due to the fact that in the uneven resource requirements, the DCA scheme attains more utilization of resources by assigning resources to the APs that actually need resources. In general, there is a trade off between the QoS, the implementation complexity of the channel allocation algorithms, and the spectrum utilization efficiency [6]. Therefore, the FCA scheme becomes superior at high offered traffic load, especially in case of uniform traffic situation and DCA scheme performs better in case of uneven traffic loads. Most of the previously developed DCA schemes have involved PER and SINR for taking the channel allocation decision. As a result the computational complexities of those algorithms were either exponential or too high. Whereas by considering the utilization as the decision factor, the computational complexity of the developed algorithm has been reduced to $O\left(n^{2} \cdot m\right)+O\left(k \cdot n^{2}\right)$ and it works better than the FCA scheme with the optimal fixed reuse partitioning.

The further improvements of this algorithm can be done by prioritizing traffic. In this regard, the APs should provide individual information of utilization of resources both for high and low priority traffics. After gaining the full knowledge about the traffic priorities of the individual APs, the APC will assign subchan- 
nels according to that knowledge. Another improvement can be done by using adaptive reuse threshold for the decision concerning the reuse of resources. In this regard, the APC can trace the modulation schemes that the APs use in data transmission and can set the reuse threshold according to the modulation schemes or PHY mode.

\section{Acknowledgement}

We would like to express our sincere gratitude to Prof. Bernhard Walke for the fruitful discussions towards the success of this work. This project has been jointly funded by Siemens AG, Munich, Germany and Chair of Communication Networks, RWTH Aachen University, Germany.

\section{References}

1. Bettstetter, C: Mobility modeling in wireless networks: categorization, smooth movement, and border effects. ACM SIGMOBILE Mobile Computing and Communications Review. 5 (2001), 55-66

2. Cheong, S., Cheng, W., Letaief K.: Multiuser OFDM with Adaptive Subcarrier, Bit, and Power Allocation. IEEE Journal on Selected Areas in Communications. 7 (1999), 1747-1758

3. Choi, M., Hanzo, B.J., Munster L., Keller, T.: OFDM and MC-CDMA for Broadband Multi-User Communications, WLANs and Broadcasting. Wiley, United Kingdom. (2003)

4. Fischer, W., Meier-Hellstern, V.: The Markov-modulated Poisson process (MMPP) cookbook. Performance Evaluation. 18 (1993), 149-171

5. Furukawa, H., Akaiwa, Y.: Self Organized Reuse Partitioning, Dynamic Channel Assignment Method in Cellular systems. Proceedings of the 43rd IEEE VTC. (1993), 524-527

6. Katzela, I., Naghshineh M.: Channel Assignment Schemes for Cellular Mobile Telecommunication Systems. IEEE Personal Communications, 3 (1996), 10-31

7. Kim, I., Lee, H.: On the use of Linear Programming for Dynamic Subchannel and bit Allocation in Multiuser OFDM. IEEE Global Telecommunica tions Conference. 6 (2001), 3648-3652

8. Koffman, I., Roman, V.: Broadband Wireless Access Solutions based on OFDM Access in IEEE 802.16. IEEE Communication Magazine. 40 (2002), 96-103

9. NS-2: http://www.isi.edu/nsnam/ns/NS-2

10. Org, C.G., Schreiber,F.: The RESTART/LRE Method for Rare Event Simulation. In Proceedings of the Winter Simulation Conference. (1996), 390-400

11. Pietrzyk S., Janssen G.: Multiuser Subcarrier Allocation for QoS Provision in OFDMA Systems. Proc. VTC. 2 (2002), 1077-1081

12. Tuerke, U.: Centralized Dynamic Channel Allocation in HiperLAN/2. Diploma thesis, ComNets RWTH Aachen University (September 2000)

13. Roy, B.: Dynamic Subchannel Allocation in a Multi-cellular OFDMA System based on Interference Measurement and Traffic Situation. Master Thesis, Comnets, RWTH Aachen University (December 2005)

14. Yin, H., Liu, H.: An Afficient Multiuser Loading Algorithm for OFDM-based Broadband Wireless Systems. IEEE Global Telecommunications Conference. 1 (2000), 103-107 